\title{
Electroplated Gold in Microwave Integrated Circuits
}

\author{
ITS APPLICATION TO CIRCUIT PRODUCTION
}

\author{
I.R. Christie and W. Mazur \\ GEC Research Limited, Hirst Research Centre, Wembley, Middlesex, United Kingdom
}

\begin{abstract}
The number of microwave integrated circuit substrates required for military, aerospace and satellite purposes is growing steadily. The conductive interconnections on these substrates must be of very pure gold. In this article, aprocess for producing such gold interconnections on substrates, which bas been found reliable and adaptable to a wide range of substrate materials, is outlined. Particular attention is given to the critical gold-electrodeposition stage.
\end{abstract}

The use of the printed circuit board within the electronics industry is well known, with applications throughout the full range of electronic equipment. The board acts as the conductor track with suitable spaces to interconnect the passive and active components required to produce a functional sub-assembly. At high frequencies conventional materials for circuit boards, e.g. copper and organic resins, become less desirable due to unavoidable signal loss and distortion caused by the poor dielectric properties of the organic substrate materials.

To reduce signal distortion and loss, it is necessary to use substrate materials of a high dielectric constant, and this property is best provided by inorganic substrates. By far the most common substrate material employed at microwave frequencies is polycrystalline alumina upon which microwave integrated circuits, or MIC's, are produced by suitable metallisation processes. Other materials also used as substrates are single crystal quartz and sapphire, but the costs of these substrates prohibit their use except for specialist applications.

The selection of the conductor material is of equal importance. In addition to the obvious need for good electrical conductivity, it is also necessary to select a material which will withstand corrosive attack without significant surface oxide formation, and can be readily bonded and deposited onto the substrate, in a uniform and economic manner, at high deposit thicknesses of up to $20 \mu \mathrm{m}$.

Of all the candidate materials, only gold offers these desirable properties and it is used almost exclusively in MIC fabrication.

The purpose of this article is to describe the process and control necessary to electrodeposit gold for MIC production.

\section{Process Outline}

Before describing the gold electrodeposition process in detail, it is useful first to consider the complete fabrication process in outline. The outline will describe procedures for alumina substrates, but only minot modifications are necessary for alternative substrate materials.

\section{Cutting and Shaping the Substrate}

The substrate consists of high purity alumina which is cut to shape with precision cutting equipment. Substrates vary considerably in overall dimensions, depending on the final application, but are typically of the order of $20 \times 40 \times 2 \mathrm{~mm}$. After cutting, the two major surfaces are lapped to final dimensions and carefully cleaned using ultrasonic techniques to remove alumina debris and lapping compounds.

\section{Preliminary Metallisation}

The substrate needs to be rendered conductive prior to gold electroplating; this process is usually carried out by vacuum metallisation. The favoured technique is magnetron sputtering in a two stage process.

The substrate is first cleaned under vacuum by ion bombardment, and a thin ( $50 \mathrm{~nm}$ ) layer of nickel-chromium alloy is deposited. This step is critical in providing the initial adhesion to the substrate. It is thought that some chemical reaction occurs between the chromium and the freshly cleaned alumina to form an alumino-chromate layer under the evaporation conditions.

A second layer, consisting of pure gold ( 99.9 per cent), is deposited over this nickel-chromium film without removing the substrate from the vacuum chamber. This gold film protects the nickel chromium underlayer from oxidation on removal from the vacuum chamber, and gives a good surface for the subsequent gold electrodeposition. To reduce the possibility of oxidation of the nickel chromium sub-layer after vacuum metallisation, the substrates are electroplated to full gold thickness with the minimum of delay.

\section{Electroplating}

The electroplating step is carried out in a near neutral cyanide electrolyte capable of depositing a pure 99.9 per cent gold $=$ To avoid entrapment of metallic impurities in the electrodeposit no grain refiners are used. The thickness of the gold deposit varies, depending on circuit tequirements, but usually falls between the ranges of 5 to $20 \mu \mathrm{m}$. At this stage, it is possible to halt production for inspection and testing without fear of affecting the adhesion. It is common practice to build up stocks of fully metallised substrates against further orders.

\section{Patterning of the Circuit}

The conductor pattern is usually required only on one side of the substrate, but for many applications the reverse side is also metallised to ensure adequate bonding to the mounting unit, and to facilitate heat removal.

Photo resist material, UV sensitive, is applied by spinning techniques to the surface, and exposed to ultra-violet light via a mask which defines the resist pattern. 


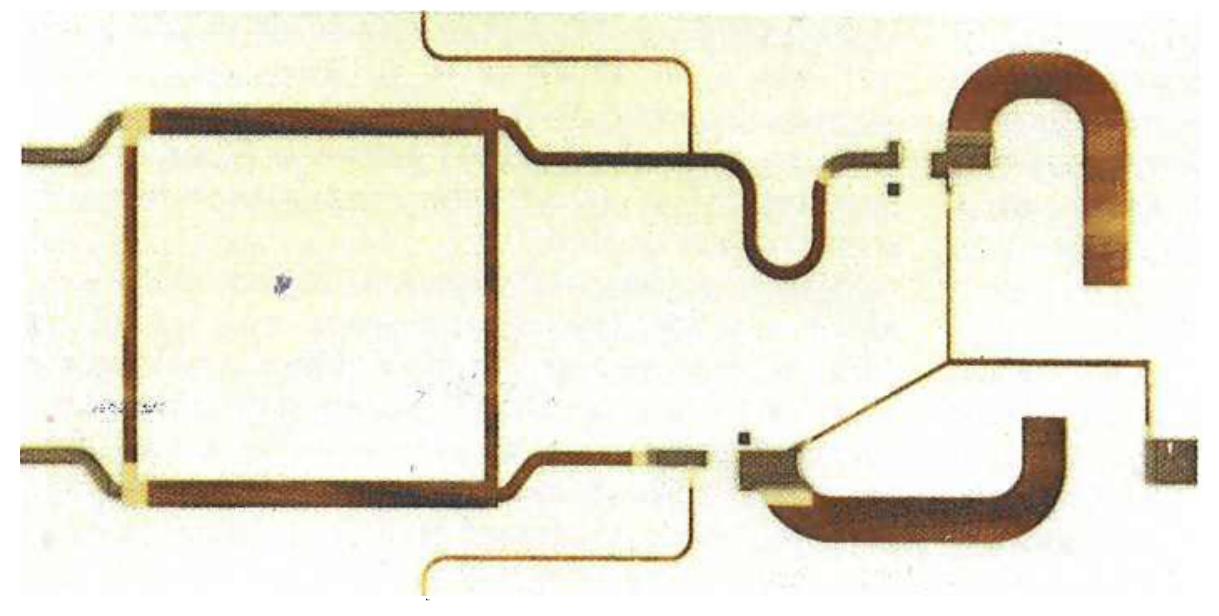

Unexposed resist is removed in suitable solvents and the unmasked gold and nickelchromium removed by chemical etching. The exposed resist prevents attack by the reagents leaving the desired tracks and patterns.

After cleaning and inspection to ensure that all uncoated metal has been removed, the UV hardened resist is removed. Examples of the final component are illustrated in Figures 1 and 2.

Fig. 1 S band balance-mixer gold on alumina substrate.

Fig. 2 Substrates other than alumina also used in MIC fabrication:

(Top left) $Q$ band front end on lapped quartz. (Bottom left) $\mathbf{S}$ band amplifier on sapphire.

(Centre) Rat race coupler for beam lead diodes on quartz. (Right) Amplifier circuits on glass with thin film resistors.

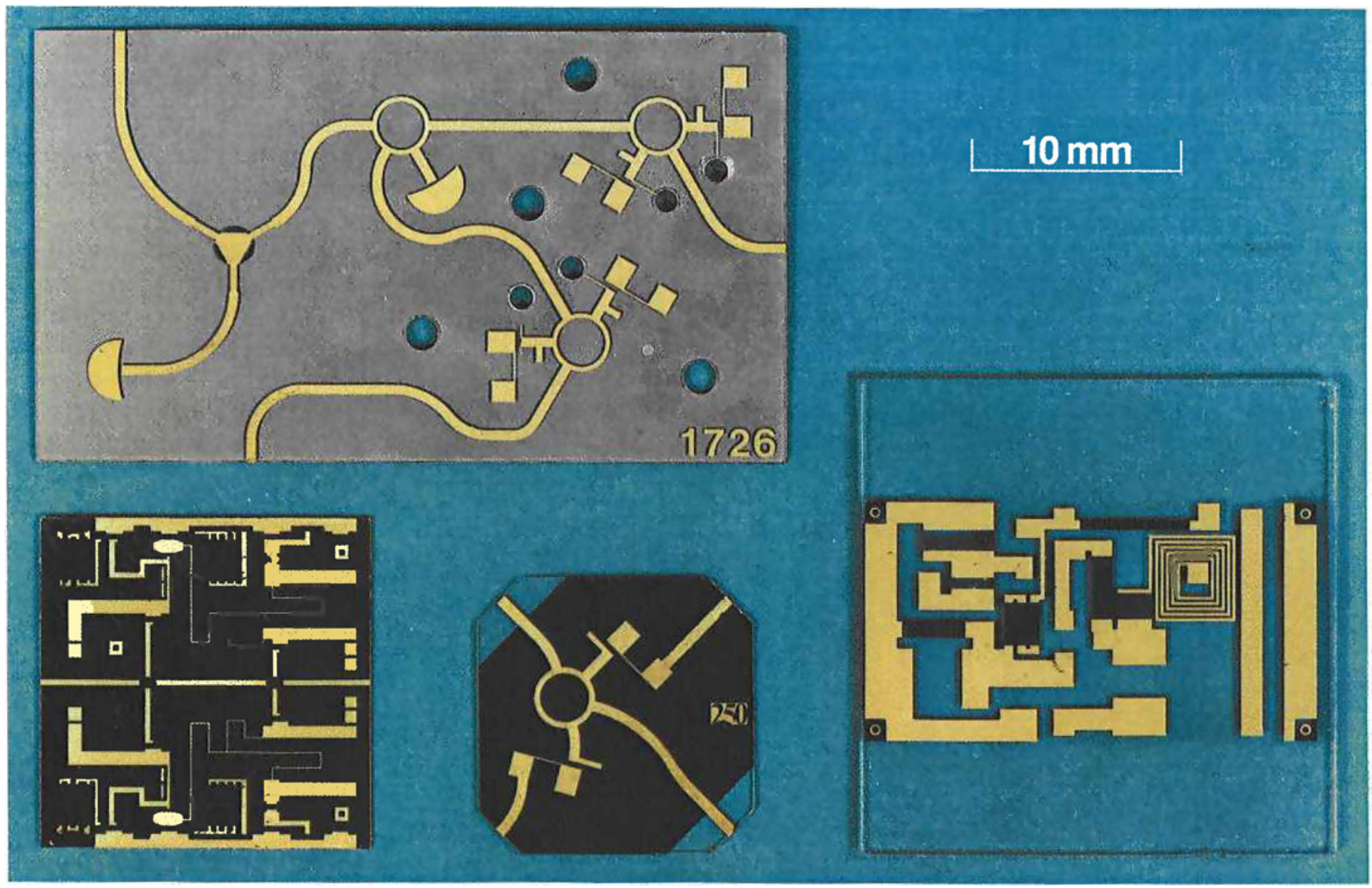

GoldBull., 1986, 19, (2) 


\section{Solder Pads}

In some cases further metallisation is required on solder pads. In these cases the conductor pattern is remasked except for the pads. Electroless nickel (a diffusion barrier) and electroless copper (an aid to subsequent soldering) are then deposited over the selected regions only.

\section{Details of Gold Electrodeposition Process}

The above section briefly outlines the whole process of MIC manufacture. The processes and control necessary in the gold electrodeposition stage will now be discussed in detail.

For this application it is necessary to produce a very high purity gold containing no brighteners or metallic grain refiners. In this way a gold of high density and electrical conductivity can be obtained to maximise signal transmission. It is also necessary to produce a smooth gold surface onto which the resist can be applied uniformly.

Some proprietary baths for production of high purity golds, whilst achieving the required purity, yield a grain structure which is characterised by angular facets when examined at high magnification. Such structures must be avoided for high frequency transmission, as the sharp irregular grains can lead to signal distortion. In addition, the coarse surface structure of the alumina substrates must not be enhanced by the gold electrodeposit, and good levelling and throwing power characteristics are desirable features. A further requirement is that the electrolyte should be effective at near neutral, or slightly acidic $\mathrm{pH}$, to avoid possible slow dissolution of unprotected alumina areas.

\section{Plating Baths}

To achieve these characteristics, two bath formulations have been developed which produce deposits with the best compromise between the desired properties. One formulation is based on sodium gold cyanide, which produces a somewhat lower stress level than an alternative potassium gold cyanide based formulation.

Because of the stringent requirements, it is more appropriate to prepare the electrolytes in-house rather than utilise near equivalent proprietary formulations. This allows usage of chemicals for electrolyte make-up of the highest commercially available purity, and, with a full knowledge of the formulation, a better understanding of the chemistry of the deposition process.

The two preferred formulations are given in Table I. As can be seen, they are both simple to prepare and can be made up from readily available chemicals.

Preparation of the gold electrolyte must nevertheless be carried out with care. After dissolution of all salts except the gold salt, the bath is carbon treated to remove organic impurities which may be present in the materials used. Carbon treated deionised water is used in the make-up and service to adjust the volume of the electrolyte. The gold salts are added immediately prior to initial use, and plated coupons are prepared for efficiency measurements and trace metal analysis.

\section{Bath Control and Operation}

It is necessary to avoid contamination of the electrolyte by airborne particles or extraneous metals. To achieve the former, the gold plating operation is carried out under fume hoods using filtered air, within the plating room. To avoid metallic contamination, the cathode and anode bars, and contacts, are heavily gold plated. The condition of the gold plate is inspected regularly and the plate is replaced if it shows any sign of wear. The bath is continuously filtered through a $2 \mu \mathrm{m}$ filter core, and oil-free air is used as the principal means of agitation.

Maintenance of the electrolyte is carried out daily. The amount of gold to be added is assessed from a record of the ampere-minutes of charge passed through the bath the previous day. Analysis of constituents is carried out weekly, while minor additions of gold are made to counteract any daily variation.

The electrolysis slowly produces unwanted organic material by electropolymerisation which, if allowed to build up, can result in deterioration of the properties of the electrodeposit. This is monitored by Hull cell tests carried out at $0.5 \mathrm{~A}$ and at operating temperature.

From an electrolyte in good condition, a good quality deposit should be obtained over the range $0.5-5 \mathrm{~mA} / \mathrm{cm}^{2}$. If the range is

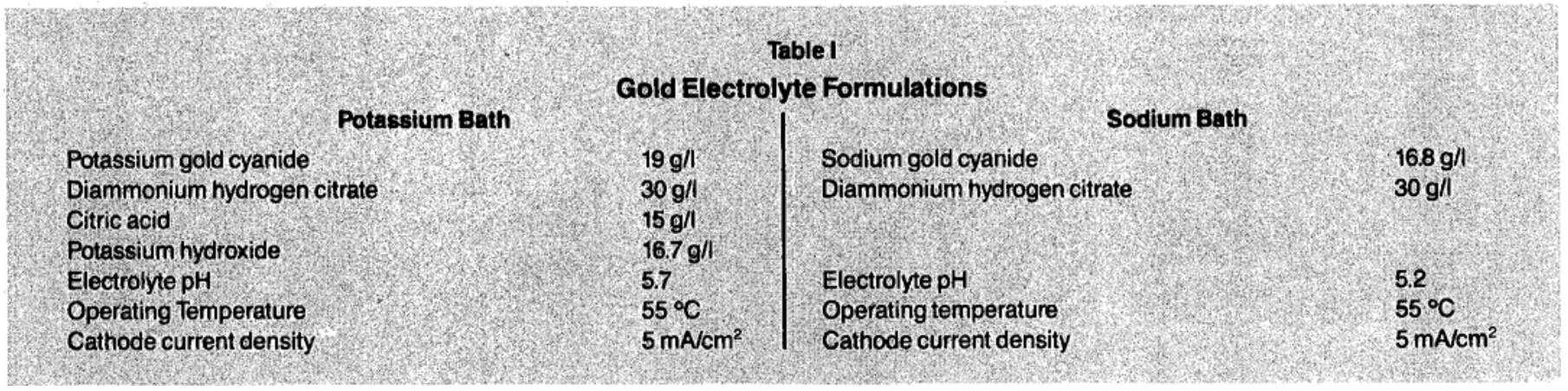


less than this, the bath is treated to remove impurities by peroxide addition and carbon treatment.

After peroxide treatment the efficiency of gold deposition falls, and a combination of electrolysis and holding at operating temperature is required to bring the electrolyte back to full deposition efficiency.

After carbon treatment the gold content is analysed, as some gold is usually removed by adsorption onto the carbon.

The operational life of these baths is typically about two years. The life is usually not dependent on usage intensity, rather it is limited by the accidental build up of trace metallic impurities in solution, usually derived from the plating salts - especially the gold cyanide complexes.

The major contaminant in gold cyanide complexes is iron, and levels in the gold electrodeposits approaching 0.01 per cent are considered unacceptable. There is no easy method for the removal of this impurity, and the bath is discarded when an unacceptably high iron figure is approached.

\section{Distribution of Gold}

Due to the dimensions of the substrate, the electrodeposit thickness at the edges and corners of the circuit will be considerably greater than in the centre. This gives rise to two problems: (1) More gold deposit is required to produce the minimum thickness at the centre, hence increasing plating time and cost. (2) The uneven thickness of gold can result in an increase of etching time required during conductor track fabrication. This, in turn, can lead to some undercutting of the protected gold track, causing an increase in the electrical resistivity.

It must be remembered that only simple gold formulations are utilised, and that the specification for the ultimate products preclude the use of grain refiners or levelling agents.

Trials were therefore undertaken to study the distribution of gold across the array of substrates during the gold electrodeposition process.

The set-up adopted for processing is given in outline form in Figure 3.
Fig. 3 Plating cell assembly to determine the distribution of gold deposit across the substrates. Screen and robbing wire used to shield lower edge of substrates.

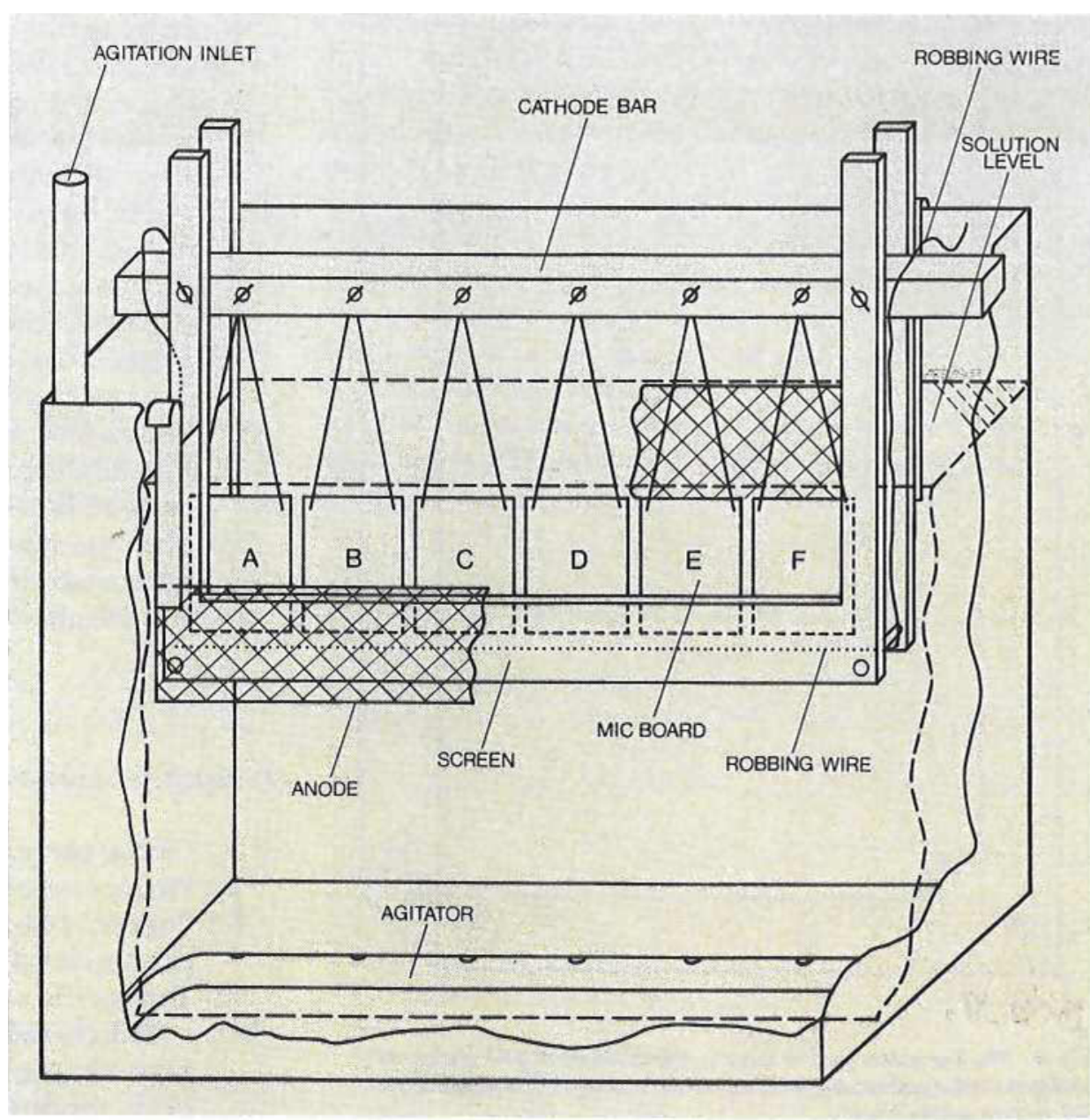


Several configurations of jigging arrangements were tried. However, it was found that if more than one row of substrates was processed at a time, those centrally-placed had deposits well below the thickness on adjacent substrates.

Thickness measurements were carried out on the gold deposits, using beta backscatter techniques, in the centre and edges of $4.0 \times 6.7 \times 0.2 \mathrm{~cm}$ circuits positioned as indicated in Figure 4 .

To obtain accurate thickness standards for these measurements it proved necessary to manufacture coupons plated on alumina substrates. The thickness figures for these were determined from microsections made through the spots at which the betascope readings were taken. A set of standards was produced for daily calibtation tests of the thickness measurement equipment.

Using a single row of circuits without shields and with air agitation only still gave a wide distribution of gold thickness as is

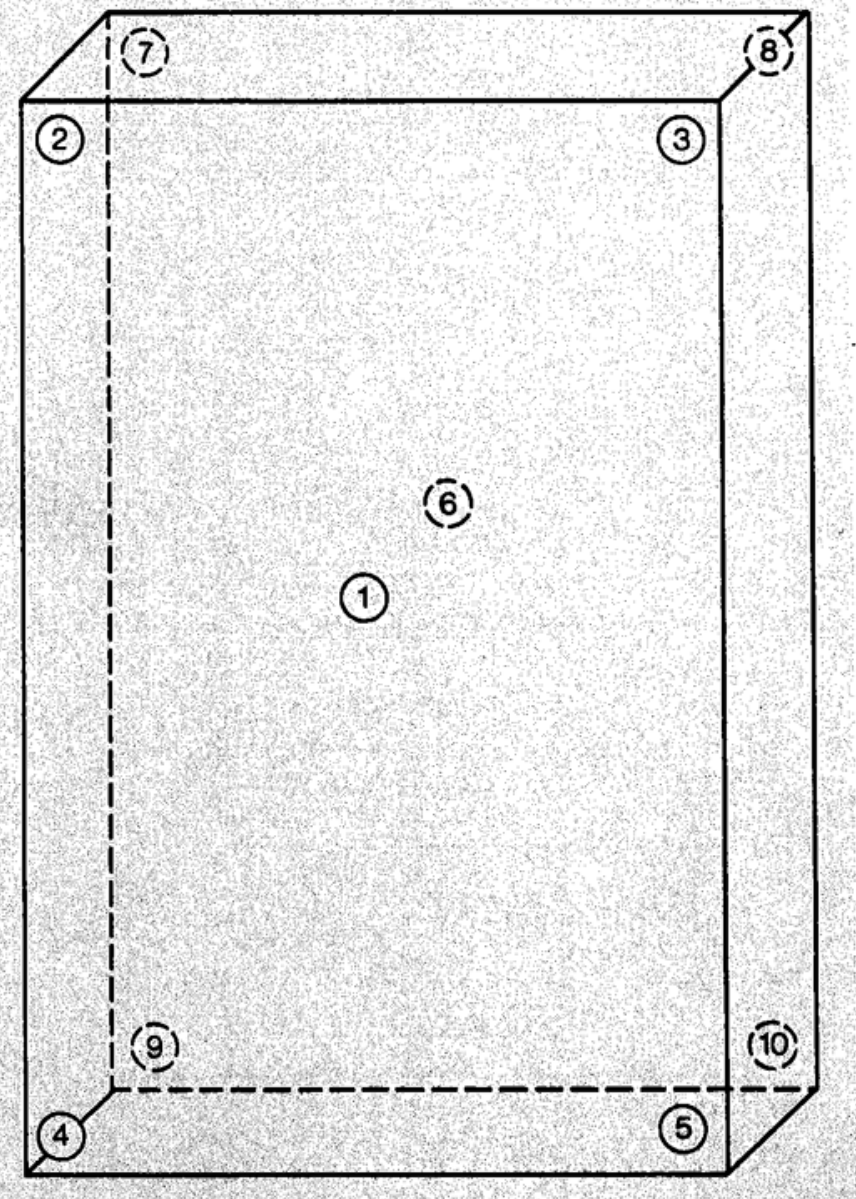

Fig. 4 The five points used to measure the thickness of gold plating on standard alumina substrates, thickness $0.2 \mathrm{~cm}$ and surface dimensions either $4.0 \times 6.7 \mathrm{~cm}$ or $3.8 \times 7.6 \mathrm{~cm}$. indicated in Table II. A maximum deposit thickness ratio(see Table II) of 1:3.2 was recorded. Using a perspex screen to shield the lower edge of the substrate and a fine gold-plated wire robber, positioned as indicated in Figure 3, reduced this ratio to approximately 1:2.0. This was considered satisfactory for the etching process and at the same time was instrumental in reducing deposition times by some 35 per cent. The effects of optimum shielding on a larger substrate $(3.8 \mathrm{~cm} \times 7.6 \mathrm{~cm})$ improve distribution further.

\section{Faults and Their Correction}

Several faults have been detected during processing of batches of circuits and this has led to a number of observations on the control parameters required to achieve a consistently satisfactory product. The more important faults, together with their causes and methods for correction, are listed below and opposite.

\section{Poor Adhesion of Metal to the Alumina Substrates}

(a) No adhesion between the nickel/chromium layer and the substrate. This can be caused by poor vacuum conditions, insufficient pre-vacuum cleaning or moisture in the vacuum system. It is remedied by inspecting and correcting the vacuum chamber conditions.

(b) Poor adhesion between the nickel/chromium and evaporated gold layers. This is due to either contamination of the vacuum systems as in (a), or to the substrates being left in air for a considerable time between evaporation goldcoating and electroplating.

(c) Evaporated gold/electroplated gold adhesion failure. This is due to either vacuum pump oil contamination during the vacuum let down cycle, or to non-specific contamination from handling. The presence of oil can usually be observed by a water break test. No globulation of water should occur when the substrate is immersed in distilled water and subsequently drained. This fault can be remedied by solvent and cathodic alkaline cleaning before gold electroplating.

\section{Rough Gold Electrodeposit}

(a) Circuits plated at too high a current density.

(b) Particle contamination of the gold electrolyte.

(c) Organic contamination or build up by cyanide polymerisation.

(d) Trace metals, e.g. zinc; contaminating the electrolyte.

Both (b) and (c) can be corrected by carbon treatment following a peroxide addition. However, if the fault is caused by (d), the electrolyte must be discarded. 


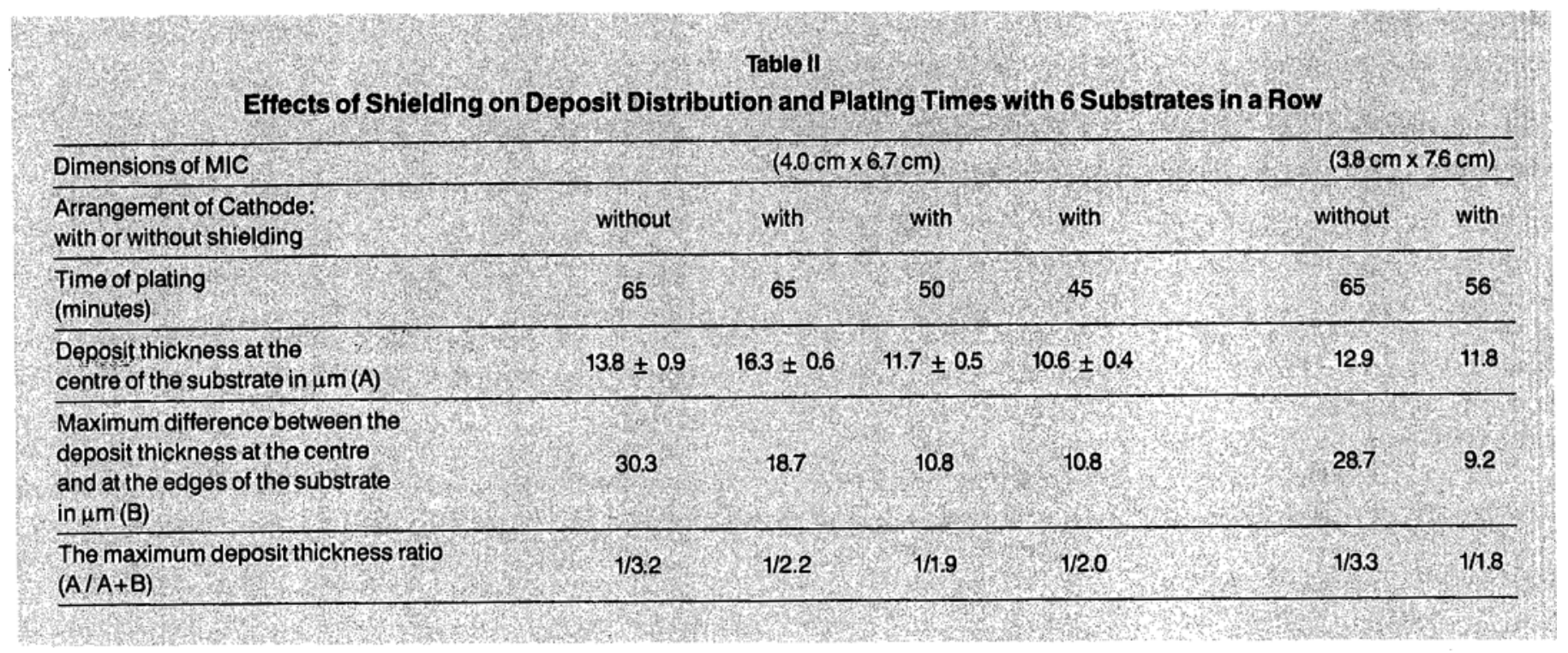

\section{Poor Gold Distribution}

(a) Air agitation too great, causing the bottom edge of the substrate to come close to the anode.

(b) Bath shields incorrectly positioned for the specific circuit dimensions.

\section{Low Gold Thickness}

(a) Current too low, or time in the electroplating tank too short.

(b) Bath requires carbon treatment. This can be checked by the Hull Cell Test and the Efficiency Test.

(c) Surface of the substrate exceptionally rough. This can cause a significant increase in real surface area and therefore a deviation from the standardisation procedures.
The above points illustrate the most significant causes of poor quality or sub-standard thickness which have been observed during processing. It must be emphasised that for consistent quality, great attention to detail is required in the operation. The two most critical areas are the vacuum deposition processes and the control of the gold plating electrolyte.

\section{Conclusions}

The process of gold deposition used in the production of microwave integrated-circuit substrates has been outlined with particular reference to the gold electrodeposition stage. The increasing demand for circuits processed by this method indicates that this technology has now reached the stage of making a significant contribution to the design and construction of advanced communication systems. 